http://jmscr.igmpublication.org/home/ ISSN (e)-2347-176x ISSN (p) 2455-0450 crossref DOI: https://dx.doi.org/10.18535/jmscr/v9i4.05

\title{
A Rare Case of Gastric Trichobezoar with Immune Thrombocytopenic Purpura and Iron Deficiency Anemia
}

\author{
Authors \\ Dr Nishant Lal ${ }^{1}$, Dr Aiswarya R Pillai ${ }^{2}$, Dr M. Vetharatnam ${ }^{3}$, Dr Shafy Ali Khan ${ }^{4}$ \\ ${ }^{1}$ Senior Resident, Dept of General Surgery, KIMS Hospital, Trivandrum, Kerala \\ ${ }^{2}$ Resident, Dept of General Surgery, KIMS Hospital, Trivandrum, Kerala \\ ${ }^{3}$ Registrar, Dept of Neurosurgery, KIMS Hospital, Trivandrum, Kerala \\ ${ }^{4}$ Consultant, Dept of General Surgery, KIMS Hospital, Trivandrum, Kerala \\ Corresponding Author \\ Dr Nishant Lal
}

\begin{abstract}
'Bezoar' is a hard indigestible mass found in the gastrointestinal tract. Most of them are trichobezoars found commonly in children. In adolescent females, it is found to be associated with psychiatric disorders like trichotillomania (hair pulling) and trichophagia (hair eating). This is a rare case of gastric trichobezoar associated with Immune Thrombocytopenic Purpura (ITP) and Iron Deficiency Anemia (IDA) in a 15 year old female.

Keywords: Trichobezoar, Trichophagia, Trichotillomania. Immune Thrombocytopenic Purpura, Iron Deficiency Anemia.
\end{abstract}

\section{Introduction}

Trichobezoar is a rare condition where a mass of hair is found within the stomach or duodenum. It occurs following ingestion of hair from head, dolls or brushes which increases in size following accumulation of non absorbable fibers, food materials rich in cellulose, cotton matted together by protein, mucus and pectin. It can present as an asymptomatic abdominal mass in early stages or with features of intestinal obstruction or perforation. Most cases are attributed to psychiatric disorders. We report a girl, who had severe IDA that may have been the cause of PICA. Medical evaluation led to a diagnosis of ITP as possible cause of severe anemia.

\section{Case Report}

15 year old girl with no previous medical illness, presented with history of heavy menstrual bleed after attaining menarche 2 years ago. Her periods were regular with 40 day cycles and normal flow lasting 4-5 days. For the past 3 months, she had loss of appetite and loss of weight. She also had recurrent vomiting for which she was evaluated at local hospital where ultrasonography of her abdomen showed an abnormal heterogenous gas pattern in the stomach and hence was referred to our center for further evaluation.

On clinical examination, she was found to be pale, thin built with patches of alopecia on her scalp. She had asymmetrical abdominal distension with palpable, immobile hard intra abdominal mass 


\section{JMSCR Vol||09||Issue||04||Page 26-29||April}

occupying the left hypochondrium and epigastrium extending upto the umbilicus.

Her hematological examinations revealed decreased hemoglobin $(7.8 \mathrm{~g} / \mathrm{dL})$, low platelet count $(60,000$ cells $)$, decreased ferritin $(9.1 \mathrm{ng} / \mathrm{mL})$ and iron (11microg/dL). Her bone marrow trephine biopsy showed normo cellular marrow with mild eosinophilia, decreased iron stores and megakaryocyte proliferation suggestive of ITP and IDA. She was evaluated with Oesophago gastroscopy which showed a large mass in stomach obstructing the oesophago gastric junction containing hair suggestive of trichobezoar. CT scan of the abdomen showed distended stomach with large heterogenous intra luminal mass measuring $25 \mathrm{~cm}$ x $10 \mathrm{~cm}$ extending from fundus to pylorus.

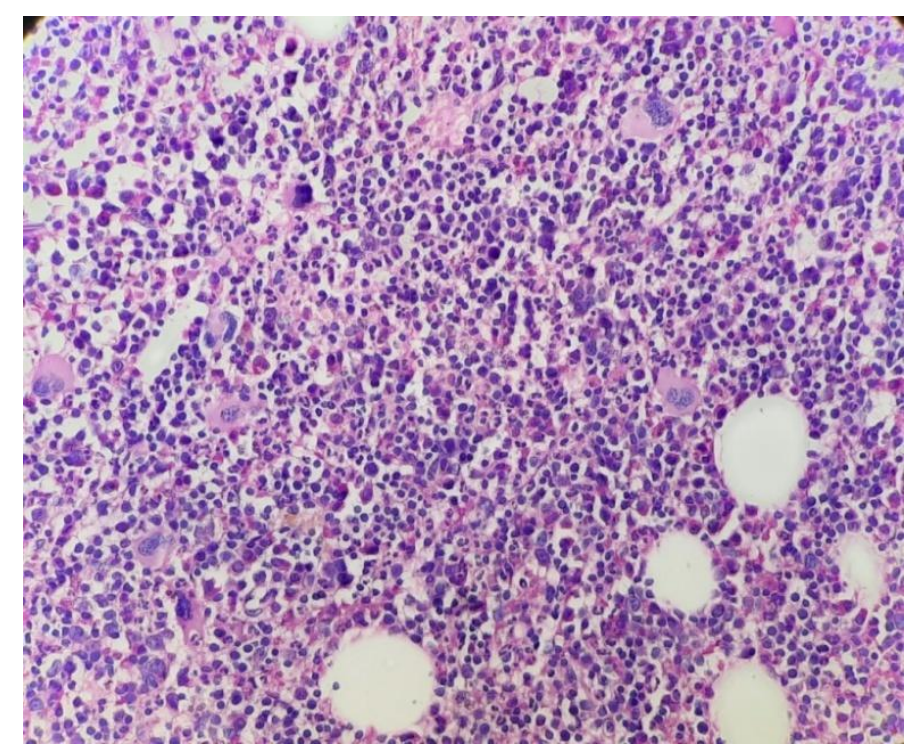

Figure I - Bone marrow trephine biopsy shows bone trabeculae with intervening normocellular marrow, mild eosinophilia, decreased iron stores (demonstrated by Perls Prussian Blue stain) and megakaryocyte proliferation suggestive of IDA and ITP.

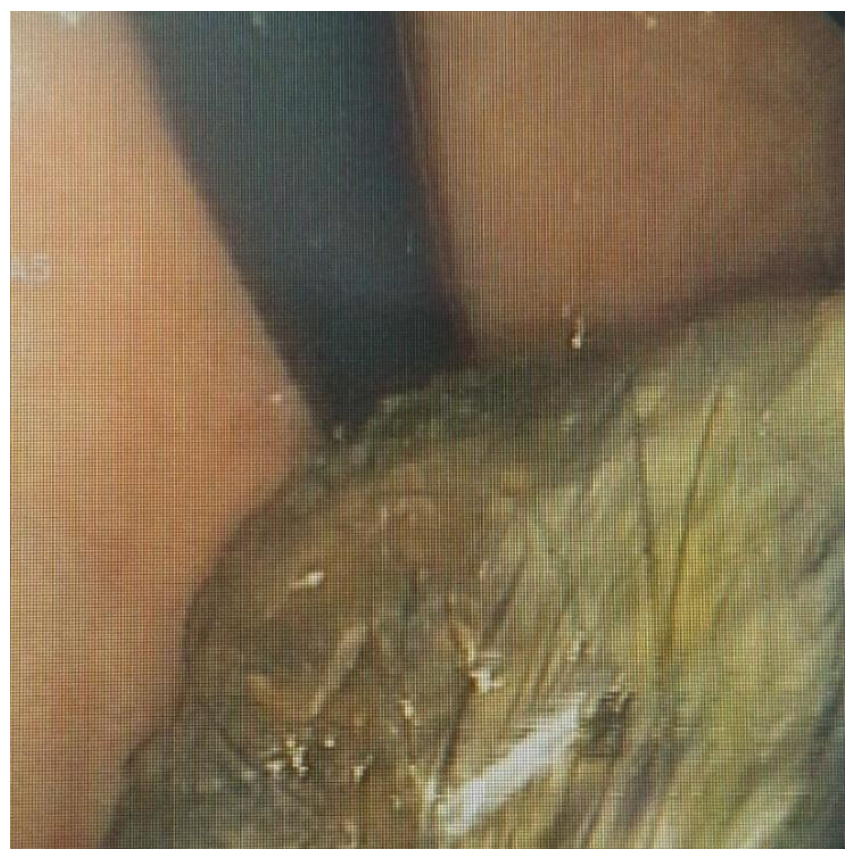

Figure II - Oesophagogastroscopy shows a large ball of hair follicles noted in the stomach suggestive of trichobezoar.

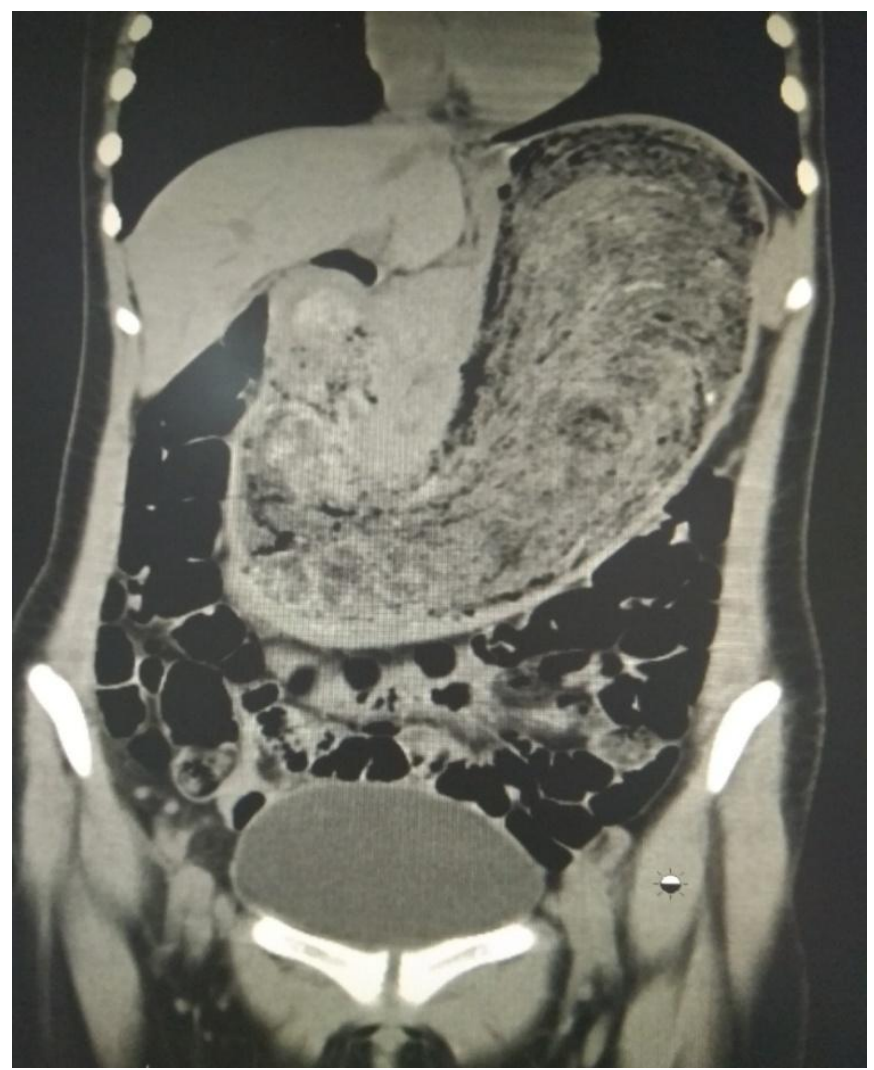

Figure III - Coronal view of abdominal CT shows dilated stomach with heterogenous mass with hyper and hypo dense contents giving a mottled appearance. The heterogenous mass is outlined by air. The mass is seen extending upto the first part of duodenum suggestive of trichobezoar. 
She was treated medically to optimize anemia and thrombocytopenia. Her preoperative hemoglobin and platelet counts were $12.5 \mathrm{~g} / \mathrm{dL}$ and 79,000 cells respectively. At laparotomy, a large lump occupying the entire stomach was noted. The trichobezoar measuring $25 \mathrm{~cm}$ x $10 \mathrm{~cm}$ and weighing 2160 grams was removed in toto. The biopsy taken from edge of the ulcer was reported as acute - on - chronic gastritis. There was no extension of bezoar into the duodenum. The girl had an uneventful postoperative recovery. She progressed to normal feeds by $5^{\text {th }}$ post operative day. She is on follow up for management of ITP and IDA.

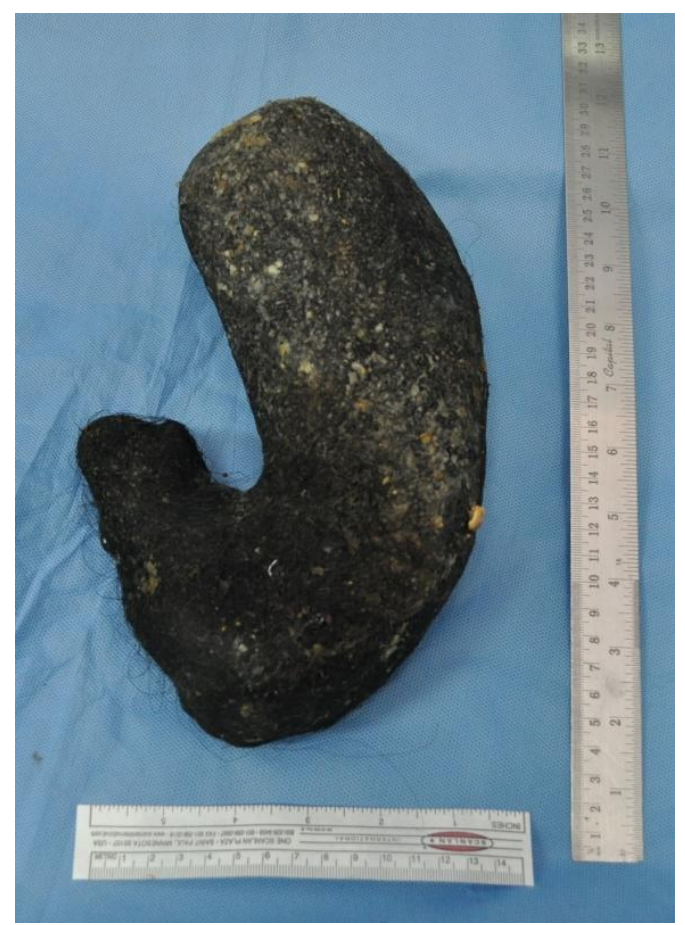

Figure IV - Gross specimen of trichobezoar removed at laparotomy.

\section{Discussion}

Trichobezoar is commonly associated with psychiatric conditions, though it can even affect healthy individuals ${ }^{[1]}$. The incidence of trichotillomania is approximately 1 in 2000 among whom $30 \%$ suffer from trichophagia; only $1 \%$ of those develop trichobezoar ${ }^{[2]}$. According to DSM-V (Diagnostic and Statistical Manual of Mental Disorders), PICA is characterized by an appetite of non nutritive substances which includes hair (trichophagia) also. Although PICA has been described in IDA, dramatic outcomes like bezoar are uncommon ${ }^{[3]}$. The case we report highlights the need to look for medical conditions before labeling psychiatric conditions. In this case ITP may have caused silent blood losses leading to severe IDA and PICA. However, IDA and malabsorption can also arise as a complication of trichobezoar $^{[4]}$. Only 3 cases of trichophagia precipitated by severe IDA have been reported yet $[5,6,7]$. The management of trichophagia involves psychotherapy and behavior therapy. These patients should be followed up at regular intervals to prevent recurrence ${ }^{[8]}$.

\section{Conclusion}

Trichobezoar is considered as one of the differential diagnosis in a young psychiatric female with mobile, hard, epigastric mass. The diagnosis is usually made with the help of endoscopy and imaging. Management involves surgical removal - most often by laparotomy. Trichobezoar in our patient was associated with IDA and ITP. A causal relationship may be inferred, if a behavior change is noted after treatment of anemia. Patients with trichobezoar should be followed up regularly as there is a high chance of recurrence.

\section{Acknowledgements}

The authors would like to thank the patient and her family for allowing publication of this case. We also extend special thanks to Department of Radiology and Department of Pathology, Kerala Institute of Medical Sciences Hospital for providing radiographic and histopathology images. All the authors have read and agreed to the final manuscript. The authors did not receive any financial support for research, authorship and publication of this article.

\section{References}

1. Coulter R, Antony MT, Bhuta P, Memon MA. Large gastric trichobezoar in a normal healthy woman: case report and review of pertinent literature. South Med J. 
2005 Oct;98(10):1042-4. doi:

10.1097/01.smj.0000182175.55032.4a.

PMID: 16295823.

2. Pace AM, Fearne C. Trichobezoar in a 13 year old male: a case report and review of literature. Malta Med J. 2003;15: 39-40.

3. American Psychiatry Association. In: Cortina R, editor. DSM V, the future of psychiatric diagnosis; 2014.

4. Cannalire G, Conti L, Celoni M, Grassi C, Cella A, Bensi G, Capelli P, Biasucci G. Rapunzel syndrome: an infrequent cause of severe iron deficiency anemia and abdominal pain presenting to the pediatric emergency department. BMC Pediatr. 2018 Apr 4;18(1):125. doi: 10.1186/s12887-018-1097-8. PMID: 29614986 ; PMCID: PMC5883293.

5. Larsson LT, Nivenius K, Wettrell G. Trichobezoar in a child with concomitant coeliac disease: a case report. Acta Paediatr. 2004 Feb;93(2):278-80. PMID: 15046290.

6. McCallum IJ, Van zanten C, Inam IZ, Craig W, Mahdi G, Thompson RJ. Trichobezoar in a child with undiagnosed coeliac disease. J Paediatr Child Health. 2008 Sep;44(9):524-5. doi: 10.1111/j.1440-1754.2008.01363.x.

PMID: 18928474.

7. Irastorza I, Tutau C, Vitoria JC. A trichobezoar in a child with undiagnosed celiac disease: a case report. World J Gastroenterol. 2014 Feb 7;20(5):1357-60. doi: $10.3748 /$ wjg.v20.i5.1357. PMID: 24574811 ; PMCID: PMC3921519.

8. Kırpınar I, Kocacenk T, Koçer E, Memmi N. Recurrent trichobezoar due to trichophagia: a case report. Gen Hosp Psychiatry. 2013 Jul-Aug;35(4):439-41. doi: 10.1016/j.genhosppsych.2013.01.013. Epub 2013 Mar 29. PMID: 23541805. 